\title{
The Liminal in a Diptych: A Study of Roots and the Ruminant in Bob Dylan and Kabir Suman
}

\author{
Amlan Baisya ${ }^{1} \&$ Dibyakusum Ray ${ }^{2}$ \\ ${ }^{1}$ Department of Humanities and Social Sciences, NIT Silchar. Orcid: oooo-0oo2-5966-1108. \\ Email:amlanb.1999@gmail.com \\ ${ }^{2}$ Department of Humanities and Social Sciences, NIT Silchar. Orcid id: oooo-0ooz-9537- \\ 3277. Email: dibyakusum776@gmail.com
}

Received March 4, 2017; Revised on June 2, Accepted June 12, 2017; Published June 15, 2017.

\begin{abstract}
:
This paper compares two certain sections in the musical career of Bob Dylan and Kabir Suman to look at a possible ideological heredity--1963-65 | 1993-97-- these two timelines had established Dylan and Suman in their iconic status. My argument is that there is a liminal tradition-- in four separate lyrics by the two composers-- that transcends their geo-temporal boundaries. Dylan's four songs-"Blowin' in the Wind", "Tambourine Man", "Farewell Angelina", "All I Really Want to Do" have been spiritually translated by Suman in the early to mid 9os, when he was most productive musically. These songs, amongst others, not only established Suman as an avant garde musician but also seamlessly merged with his own vision of antiestablishment and non-belonging. Dylan was writing against the imperialist capital, Suman was writing against the parliamentary Left-they both assert the same bohemianism before proceeding towards iconic stasis. Dylan, after the 6os turns towards safer, politically inert aesthetics; Suman partially removes himself from music in favor of a fledgling political career. The bohemianism or the perennial non-conformity ends for both. What is the significance of this phase? How liminal are the lyrics divided by language, time and society?
\end{abstract}

Keywords: Bob Dylan, Kabir Suman, Liminal, Roots, the Other, Translation

\section{1.o Introduction}

Although he has reportedly claimed: "Dylan was never my guru", Bengali singer and a stage performer Kabir Suman has, in the same reportage $\mathrm{i}$, attributed his meteoric rise and decadetranscending lyrics to Bob Dylan: "Dylan's simplicity, in fact, sent me back to my folk roots." My argument of treating Bob Dylan- Kabir Suman as a diptych hinged by the same liminal philosophy finds a distant ringer in the latter's otherwise cursory tribute to Dylan winning the Nobel. Two words are of significance here-- 'roots' and 'liminal'. If perceived as a return to a sentient, prelapsarian cora, the first word has volumes to tell-- from religious to political. For brevity's sake, let us assume that Suman is talking about an idealized musical heritage-- innocent, transcendental, non-binarized. Dylan himself has been described as a predominantly collage artist ${ }^{\mathrm{ii}}$, and the stylistic and thematic influence of Country, Rock n Roll, Gospel musical traditions are rife in his songs. Dylan was a cultural expatriate from rural Minnesota, and has consciously, continually distanced himself from edifying fons et origo throughout his career-- from Jewish to Christian, protest to introspection, counter culture to establishment. As Jeff Taylor notes in 'The Political (c) AesthetixMS 2016. This Open Access article is published under a Creative Commons Attribution Non-Commercial 4.0 International License (http://creativecommons.org/licenses/by-nc/4.o/), which permits non-commercial re-use, distribution, and reproduction in any medium, provided the original work is properly cited. For citation use the DOI. For commercial re-use, contact editor@rupkatha.com 
World of Bob Dylan', although deeply moved by Jack Kerouac, Dylan never wanted to be another Dean Moriaty.

What, then, might the word 'roots' signify? Irrespective of how Suman meant it, Dylan is a reminder of a musical root-- especially to the former's context-- in a more complex manner than a 'simple' throwback of the nativist tradition. This paper argues that the seemingly inconsequential ascendancy of Dylan over Suman's music (by the latter's repeated confirmation from the 9os to present) is not random, but a protraction of a liminal philosophy. It's neither here nor there-- a Les Rites de Passage iii $^{i}$ that constantly escapes ideological categorization, keeping itself abreast of establishment-oriented averments. Both refused to play by the rule of the state, both eschewed even the zeitgeist of jingoistic counter-establishment as well. Yet there is a catch. In spite of being close to Aaron Copland, Lotte Lenya or the Seeger family, Dylan, 1962-65, had an ambiguous relationship with the surging anti-imperial vogue. Dylan had equal debt to his native Yankee idealism. In fact, by the mid-196os, Dylan's brand of non-conforming euphonic had started to attract derisions from Rock aficionados as conservative, reactionary and discriminating. ${ }^{\text {iv }}$ Yet, this is also the phase that produced the most enduring legacy of the composer. Suman, on the other hand, enjoyed a similar patch of colossal influence over Bengali modern music; but unlike Dylan, his creative proximity was to a general disillusionment towards the global Left. The early 9os, especially in Bengal's context, was marked by an intellectual ennui coupled with a growing dispassion for the social Left, lumpenism and a fallow music-scene. Suman had-- through five subsequent studio albums from 1993-97-- his own brand of wistfulness that is part lament for an idealized past, and part rejection of the sterile present ${ }^{v}$ in search of what even Suman himself did not seem to know.

I hope that the nature of the hinge is becoming clear. Dylan and Suman-- early 6os and early 9os-- both non-conforming without being radical-- one jaded with the capitalistic excess, the other with the Left's morphing into an establishment. Both are cynical of the present, but unsure how to move on. Neither is rejecting the ennui, yet seems undecided of the 'right' direction. Suman has four iconic songs in this period directly influenced by Dylan's global hits. These are my case studies with some rudimentary translation from Bengali to show the thematic parallels. Probably Suman was thinking of this phase while talking about 'roots'. I call this rhizomatic relation 'liminal', because there is a clear progress from pensive rejection of a dystopic present to a renewed hope for something that is artistically obfuscated. What we have in both is a slow recognition of somebody other than 'Me'-- Angelina, the-girl-I-know, the Tambourine Man or a simple 'You'. Liminality is a rite of passage that is an itinerant continuum between two set places. Dylan and Suman have started their journey from a scenario of anti-establishment, and have morphed into wizened agents of establishment themselves (Dylan has been a Christian, star performer, actor and Nobel Laureate; Suman a preacher, actor, TV show host, National award winner and politician). But the liminal phase is still regarded as the most influential period for both. My argument is a close pondering over the lyrics that might reveal why, and finally bring the panels of a diptych closer to form a meta-picture that transcended geo-temporal barriers.

\section{2.o The Temporal Shift}

In this section, I will look at the lyrics of Dylan and Suman in an episodic fashion-- more thematic than it is chronological. The span, after all, is short-- less than half a decade, and it is safe to assume that the liminal tendency is homogeneous in the songs composed at this time. I start from 'Blowin' in the Wind', recorded on July 9, 1962, and proceed towards 'All I Really Want 
to Do' (1964) and two additional texts in between -- a strategy concurring to the recording dates of the Kabir Suman's versions three decades away ('Uttor-o toh Jana'(1993) to 'Chaichhi Tomar Bondhuta'(1996) and two other songs influenced by Dylan). I don't want to give the impression that this temporal shift is insignificant and/or contravened for the sake of a seamless Dylan=>Suman argument. In fact, this seam is an organic part of this article because of the previously mentioned diptych-quality of the disparate archs: one half belongs to Dylan's transition from belligerent anti-imperialism, the other initiates Suman's surreptitious critique of the parliamentary left. Put together, we perceive a cogent aesthetic evolution that starts from denouncing the Right-- forms a liminal transition which does not conform to ideology-- proceeds towards finding an established, iconic and somehow inert identity. The question is, where is the hinge that holds the parts of the cypher? If liminality is the fulcrum of this evolution, does it owe itself to the interim stretch?

\subsection{The Two Disparate Arcs: Lyrics of Dylan and the Adaptation by Suman}

The three decades of intercession is that time of germination when liberalism dressed in the attire of establishment, the left became legislative and Dylan receded into introversion by distancing himself from the liminal, eternally non-conformist polemics. In another part of the world, Kabir Suman appears as a conspicuous resumption of where Dylan left off. In the twilight of the socialist regime around the world, Suman initiates his liminal phase as free market ascends in Global proportion. The liminality of Suman, clearest through his equally iconic adaptation of Dylan's liminal songs, affirms that the gap of three decades was always gravid with a possibility of renewal-- irrespective of geo-temporal boundaries. Dylan rejects war and examines the simple, transient aspects of life-in-flux; Suman turns away from skewed humanity and bids farewell to everything 'known' or 'familiar'-- a walk into solitude. Certainly, Suman must not be the only one, and the same phenomenon must have taken place elsewhere at another time. Rehabilitating Suman in the reflected glory of Dylan was not our intention either. Dylan and Suman, by complementing each other by the latter's musical choice, only proves that the liminal theme of Dylan's songs can defy any conceivable boundary. A close look at the songs is likely to enforce this point.

\subsection{1 "Blowin' in the Wind" and "Uttor-o toh Jana": the Rhetoric of post-Protest}

'Blowin' in the Wind' was famously recorded in 1962 as part of Dylan's second album “The Freewheelin' Bob Dylan". The iconic status of the song is owed to the simple yet robust chords and lyrics that not-so-subtly provide a critique of anti-imperialism, especially the Vietnam War. The cannon balls must fly a million times, the white dove must fly untiringly and people must endure a perennial grind of slavery before the desired stasis comes. It is difficult to call this song 'hopeful', because the answer is ever-elusive-- "blowin' in the wind". The description of the grind or a pensive trudging is further clear in the imagery of a long road, the unreachable sky beyond a concrete jungle, or the habitual nonchalance for the others' despair. Dylan is certainly invested into a tangible idea of freedom from the system or an idea of alienation from an organic conception of the lived experience-- "How many years can a mountain exist/ Before it's washed to the sea?/ How many years can some people exist/Before they're allowed to be free?" The world is transient, everything that stands must fall, yet the eternal subjugation of the human defies this natural cycle. What he eschews, however, is a direct mention of events, names and places-- the song is universal, is perfectly dissociated from any delusional polemic of revolutionary change, and seems to be more interested in asking questions than answering them. Dylan's tryst with 
liminality is thus clear from his non-incumbency towards 'art as activism'.

Suman is no less non-conforming in "উত্তরও তো জানা" [Uttor-o toh Jana] ("The Answer For-granted"). While the majority of the song is heavily indebted to the same imagery invoked by Dylan, Suman seems to be even further removed from the spirit of direct activism or an invested, counter-culture and political critique. In a way, Suman is even more humanist and liminal on a greater level because his critique is of a time of disillusionment of the rhetoric of protest itself. Dylan was against imperialism, Suman is against even the anti-imperial (since the latter subsumed the former's garb). Behold a few lines:

কতটা অপচয়ের পর মানুষ চেনা যায়

প্রশ্ন গুলো সহজ আর উত্তর ও তো জানা ...

কত হাজার মরলে তবে মানবে তুমি শেষে

বদ্ড বেশি মানুষ গেছে বানের জলে ভেলে

First, Suman eschews the reference to war, because the war on humanity in his geo-political context has been surreptitious, waged by those who once defied it. Hence comes the first two quoted lines-- "Must lives be wasted to know the nature of men/the questions are simple and the answer is plain". The tone of a post-Dylan cynicism is clear in the morbid realization of failed revolution and the unmasking of the messiah in the next two lines-- "Should thousands die before you must know/ Too many have drowned in the torrent and flow". This song, recorded in 1993 for the album ইচ্ছে হল (As I Wish) is the first step towards Suman's own brand of liminal nonconformism intensified in গানওলা(The Music Vendor)(1994), itself a spiritual company of “Tambourine Man”(1965).

\subsection{2 “Tambourine Man" and "Gaanola”: A Carnival of Rust}

“Tambourine Man”, recorded for "Bringing It All Back Home” (1965), is probably Dylan's most unambiguous exploration of the liminal philosophy, "it's just escapin' on the run”. The song can be an abstruse satire: Dylan is not going anywhere, because he knows that the world is spinning around the unchanged; there is no magic in power, because the evening's empire will forever return into sand. Dylan cannot sleep, yet he cannot wake up because the realisation of the futility of existence has rendered him blind. The creative is reduced to mere repetition, the mobile only chases its shadow, and the poet is dying to escape the grind but he cannot. Dylan is clear about the self-delusional nature of control-- all the players are "circled by the circus sands", the streets are ancient and empty and its rhetoric of power is just a clown-ish self referentiality. The journey of liminality takes a decisive turn here-- "Blowin' in the Wind" is a rejection of the war mongering empire, "Tambourine Man" is a rejection of the existence sanctioned by that empire. Dylan refuses to play the game, yet he does not know of any alternate strategy. All he can do is to remain static as the world goes around in a meaningless circle, perennially waiting for 'another' last song. He is neither here nor there, unsure of where to be or whether to proceed.

Suman gives a fresh spin to the extreme pensiveness of Dylan, yet remaining just as true to the spirit of flux in গানওলা [Gaanola] (The Music Vendor). From Dylan's disillusionment of a greater, more cosmic scheme of time, Suman brings it back to his own, minute lived experience:

ছেলেবেলার সেই বেহালা বাজানো লোকটা 


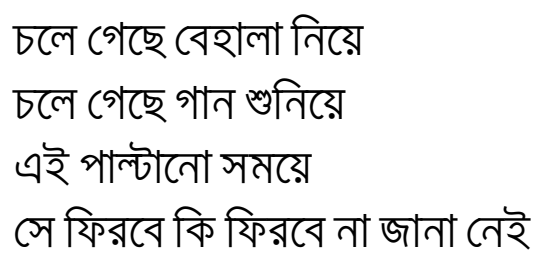

Suman is interested in the passing of a more idyllic, idealistic phase that might have succumbed to the temporal drift, but he refuses to accept the changed times: "The violin player of yore/Has parted with his violin/His song is done/And I have no clue/ If he will return in the changed time." Once again, we notice the near constant pensiveness giving birth to a Dylanesque liminal sensibility, but on a more intimate note. Suman is just as cynical of the nature of the "evenin's empire", but he has a static ideological reference point where Dylan is faced with utmost nihilism as his past. Let it be reminded that this is exactly what I am trying to argue here-- Dylan distances himself from an authoritative dogma; his liminality has a subtext of a possible initiation of something new. His despair is that of the jaded, whereas Suman's liminality is the next phase-the 'new' came, and turned out to be the 'old'. Dylan is blasé, Suman is sad. Yet liminality brings them together when the latter, just like Dylan, asks the vendor to play a last song as he has nowhere to go and nothing to do in a country of eternal gambling ("এই ফাটকাবাজির দেশে স্বপ্নের পাখি গুলো বেঁচে নেই”-- “The birds of dream are gone in this land of wager").

\subsection{3 "Farewell Angelina" and "Biday Porichita": the Liminal Merging}

The third and the fourth songs in this structure have to be in an argumentative parallel because Dylan (and Suman) signals a change in the liminal worldview, if such a thing is at all possible. My point is, from the eternal 'I know not where I stand', Dylan seems to have found his footing somewhere in the nowhere. First comes "Farewell Angelina", recorded in January, 1965, and released two and a half decades later in "The Bootleg Series, Vol. 1 - 3" (1991), although Joan Baez had already popularized the song 1966 onwards. Possibly the most complex text in our purview, "Farewell Angelina" belongs to somewhere between the empire of dust and a renewed hope in constant anti-establishment. Dylan "must go", because everything is still the same, and there is no decisive emotion that might turn the table in the already troubled relation with nothingness. "Tambourine Man"'s fatal cynicism is abundant still-- the pirates have lost their way and the neighbors treat them as circus freaks, the giants and the dwarfs continue the same absorption and re-absorption of authority and power while the innocents die. Time does not matter anymore as the hands of the clock are stolen themselves. Yet there is a change, because the "sky is trembling" and Dylan must go even if he knows the futility of moving in a circle. There is a change coming, the dystopic world of the ruler and the ruled is soon turning into ash but Dylan is not going to be a witness to the destruction. This is what I call a motif of rejection, germane especially in context of "All I Really Want to Do" (1964)-- which finally brings the circle to the next level of continuum. In "Farewell Angelina", he is still not sure of his destination, and neither is Suman. The latter-structurally and philosophically-- is also growing out of the baggage of his geo-specific sensibilities, and "বিদায় পরিচিতা" [Biday Porichita] (Farewell, the Girl I know) is a testimony to Suman's merging with Dylan's liminal philosophy. Dylan talked about a time-bomb on a short fuse in "Farewell Angelina" noting that the destruction is imminent. Suman is still more pensive. In his song, the sky is neither erupting nor angry-- just nonchalant or plane sad. There is no countdown to an explosion, just the corpses of murdered dreams and old Dylan writing songs that 
nobody will hear. Yet Suman must go too:

রাস্তায় পড়ে আছে স্বপ্নের লাশ
বাতাসে লুকোনো তার দীর্ঘশ্বাস
খুন হওয়া স্বপ্নের চোখ ঢেকে দেওয়া চাই
বিদায় পরিচিতা আকাশ বিষন্ন, তার কাছে যাই
কি হবে গান লিখে তবু লিখি গান
বয়েস হলো আমার বুড়ো হলেন ডিলান
তুমি শোনো বা না শোনো শুধু তোমাকে শোনাই
বিদায় পরিচিতা তুমি একা আমি একাই ."

"My road is fraught with aborted dreams/ I hear its sighs in the leaden breeze/ Please cover their listless eye/ Farewell, I leave for the pensive sky"-- Irrespective of a general ennui, the theme of rejection is clearer than ever in Suman's oeuvre. I hesitate to call it decisiveness, but the dogged persuasion belies a sense of an indurate mindset. The grind goes on, but the poet refuses to follow the suit, although the time of hope is yet to come: "The ballad has lost its charm, yet I sing/As old Dylan does in his faded bling/ May you listen or not, yet I sing my song/ Farewell my love, we're loners for long." This was in 1997, almost on the verge of Suman's post-liminal phase of political and religious obduracy in নিষিদ্ধ ইস্তেহার (The Forbidden Manifesto) and আদাব (The Salute)-- the two albums of Suman's initiation into Islam and parliamentary politics followed in 1998 and 2002, and it indeed seems he is writing a final elegy for the time that is lost forever.

\subsection{4 "All I Really Want to Do" and "Chaichhi Tomar Bondhuta”: A Truce with the 'Other'}

The last text of our consideration is "All I Really Want to Do", recorded in 1964 for the album "Another Side of Bob Dylan", and widely acclaimed as one of the most significant songs that Dylan wrote in the period immediately after he abandoned topical songwriting. Dylan has never been clearer in his liminal standing and neither has Suman in চাইছি তোমার বন্ধুতা [Chaichhi Tomar Bondhuta] (I Seek Your Friendship), a song heavily influenced by Dylan's version. The semblance to a diptych is not only in the spiritual bonding of the two songs, their very titles complement each other. All I really want to do is to seek an alliance with you. There are reasons-both topical and philosophical-- why this is clearly liminal, I will cite only one for the sake of brevity. The postcolonial concept of liminality is heavily indebted to a strand in continental ethics-- the 'Other'. I will avoid citing seminal works focused on this, because there are too many on a topic already unfathomably complex. The 'Other' is the one who remains on the threshold. The one who does not conform and is essentially unreadable because it is not and will never be recognizable by 'Me'. Irrespective of my best intentions and supreme magnanimity, the 'Me' is captured in a cycle of self-recognition-- whatever I see or speak are only intelligible because I decode their existence in my way. In this sense, the 'Other' is everywhere because I unfailingly alienate everything in my obstinate persuasion of recognizing everything in my own language and perception. Emmanuel Levinas' life's work, specially summarized in Entre Nous (1998), has been to offer a kind-of resolution to this conundrum, although no resolution is ever possible. To know the 'Other' is an impossibility, says Levinas, because to really know the 'Other' entails disclaiming the 'Me', which cannot happen as long as the 'Me' is alive. What is possible is a recognition, an 
innate responsibility for this ever-belligerent relation because that, comments Levinas who is also a commentator on religious ethics, is the way proper 'living' can be achieved.

Let us spare a moment for the critical arc that has brought Dylan and Suman to our present juncture. At the beginning, it was a critique of a self-indulgent time, yet both the songwriters refused to take any alternate side. The resultant indecisiveness has begotten a deep pensiveness which again is liminal as the author rejects the ennui yet does not/cannot specify the way forward. To seek the affinity of the 'Other' can hardly be called a way out, because to know, to recognize and to let it be is a more complex iteration of inwardness-- a spiritual u-turn. The world has ceased to make meaning, all that remain are fragments, to recognise this is to admit immobility-- yet this is pregnant with an ever-renewable possibility of a fresh start. This is falling in love with the moment, and to live it eternally in spite of its inevitable passing into obscurity.

I ain't lookin' to compete with you

Beat or cheat or mistreat you

Simplify you, classify you

Deny, defy or crucify you

All I really want to do

Is, baby, be friends with you.

Dylan is just as blase about his surrounding as ever, his only active persuasion is to "be friends with you". The relational aspect of a friendship is myriad. It can be everything or nothing, close or distant, passionate or formal-- yet it recognises that you are. Suman is no less partisan:

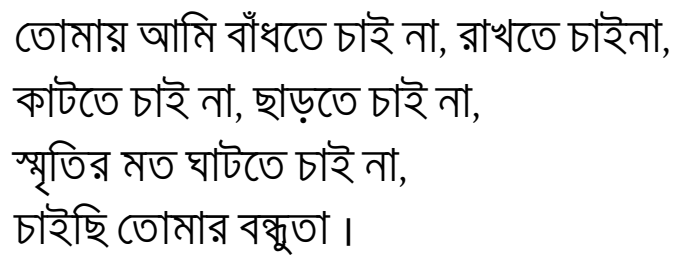

"I do not want to keep you or bind you/ Dissect you or leave you/ Rummage you like I do a memory/ All I want is your affinity" Dylan is not interested in almost anything that has a remote connection to the 'you' as a person-- not the identity, caste, creed, intentions, family or societal traits: yet the friendship is actively sought. It is clear that the 'Other' is perfectly disembodied for Dylan, it may or may not have a tangible existence but Dylan is unfazed. This brutal simplicity can be perceived as the most complex part of Dylan's liminality because of the apparent untenability of having any connection to something that does not have a body. On the other hand, this non-corporeality is symptomatic of a boundless liberty where 'I'-- in no conceivable manner-- can influence the 'Other'. Suman actually says the same in his version: “তোমায় আমি গড়তে চাই না, পড়তে চাই না” (I don't want to make you, or read you). The inefficacy of my translation that completely obfuscates the literary value of this line should not necessarily hinder the impact of such a declaration. Dylan and Suman have formed a rare camaraderie across geotemporal spaces. The former swears that he does not intend to make the other 'feel', 'see', 'be' like him, where Suman closes his song by disclaiming the readers' desperate attempt to decipher this paradoxical relationship. Dylan makes a statement, Suman strengthens it. "উটকো লোকে, এই সুযোগে, তাল মিলিয়ে/বিশ্রী কথা কাটবে জুড়বে,/নিজের জ্বালায় নিজেই পুড়বে” (The average Joe, shall take his cue/Will gravel us, with his paltry view/Shall burn within, and burn anew). Recognising 
the 'Other', not interfering with it and still be content, and therein find a possible equilibrium in a state of flux-- these are the primary traits that forever bind Dylan and Suman.

\section{3.o Conclusion}

This paper has attempted to establish a philosophical journey of Bob Dylan and Kabir Suman, through the sophomore phases of their career-- divided by decades yet having a collusion on the thematics. The journey is structured: rejection-- ennui-- acceptance-- recognition. The project, at the outset, might have seemed capricious, but a similar look at the lyrics along with the negotiation by the composers with their respective political-temporal backdrop amply reveals the complementary traits in both the arches. A parting reference to the Marilyn Diptych (1962) by Andy Warhol may summarise the innate nature of the collusion. The painting has twenty five pictures of Marilyn Monroe on each of the tablet-- one set brightly coloured, the other black and white. Read simultaneously as a meditation on life and death, youth and maturity, prominence and fading away or just the passing moments in general-- Marilyn Diptych does contain two phases of a singular icon in different perspectives. Together, however, they reflect on the transcendence of artistry and temporality. Together, Dylan and Suman, form a commentary on the sublimity of timeless artistic influence. It is also, a somewhat pensive account of the passing of an idealized creative phase.

A Note on Translation and Transliteration: All the translations and transliterations used in the paper are done by the authors unless otherwise mentioned.

\section{Endnotes}

${ }^{i}$ See "Bob Dylan's music signifies protests against injustice: Kabir Suman”, Kabir Suman, Oct 13, 2016, Hindustan Times, New Delhi, India http://www.hindustantimes.com/art-and-culture/why-bob-dylanreminds-of-bengal-kashmir-kabir-suman/story-z8PHuWoQfwXwlECDEMfHTI.html

ii See "Collage in Twentieth-century Art, Literature and Culture", Rona Cran, 2014, pg.187

iii See "Les Rites de Passage", Arnold Van Gennep, 1909, pg. ii +288

iv See "The Political World of Bob Dylan: Freedom and Justice, Power and Sin", Jeff Taylor and Chad Israelson, 2015, pg. 35

v Suman always had a troubled relationship with the then Left-government of West Bengal. In the documentary, "Free to Sing? The Music of Suman", 1996 by Sudipta Chatterjee, he directly accused the Communist Party of India(Marxist) to have intentionally disrupted his solo concerts, and sabotaged his record sales. "Look at me...I'm against establishment. I don't like our government. They are against me", says Suman at the $47^{\text {th }}$ minute of the video.

\section{Reference}

Anderson, T. (2016). The Sixties. Old Tappan: Taylor and Francis.

Baltiz, A. (2005). Globalization and Musical Culture. Acta Musicologica, 77(1), 137-150.

Bob Dylan. (2008). Choice Reviews Online, 46(04), 46-1986-46-1986. http://dx.doi.org/10.5860/choice.461986

Campbell, M. Popular music in America.

Cavell, S. (2001). Music and culture. Impressions of revolution. The Musical Quarterly, 85(2), 264-273. http://dx.doi.org/10.1093/mq/85.2.264

Chatterjee, S. (1996). Free to Sing? The Music of Suman. Retrieved from https://www.youtube.com/watch?v=CCpRaiVhUCs 
Cran, R. (2014). COLLAGE IN TWENTIETH-CENTURY ART, LITERATURE, AND CULTURE (1st ed., p. 187).

[S.l.]: Routledge.

Cumming, J. (2016). Dialectic of Enlightenment. Verso.

Das, T. (2004). Bangla Band: Adiparbo (1st ed.). Calcutta: Ultoroth.

DeSouza, L. (2001). Boys who rocked the world. Hillsboro, Or.: Beyond Words Pub.

Dylan goes electric!: Newport, Seeger, Dylan, and the night that split the sixties. (2015). Choice Reviews Online, 53(05), 53-2141-53-2141. http://dx.doi.org/10.5860/choice.194251

Dylan, B. (2006). Lyrics 1962-2001. London: Simon \& Schuster.

Dylan, B. \& Williams, C. (1993). Bob Dylan. London: Omnibus Press.Dylan, Bob. (2004). Chronicles: Volume one. New York, Toronto, London, Sydney: Simon and Schuster.

Gitlin, T. (1993). The sixties. New York: Bantam Books.

Gottlieb, R. \& Kimball, R. (200o). Reading lyrics. New York: Pantheon Books.

Isherwood, C. \& Bucknell, K. (2010). The sixties diaries. London: Chatto \& Windus.

Levinas, E. (2006). Entre Nous (1st ed.). Edinburgh: A\&C Black.

Moore, A. (2008). Analyzing popular music. Cambridge [u.a.]: Cambridge Univ. Press.

Roszak, T. (1995). The making of a counter culture. Berkeley: University of California Press.

Roy, S. (2001). Understanding Popular Music (1st ed.). London: Routledge.

Siske, J. (1989). Reading the Popular (1st ed.). Boston: Unwin Hyman.

Sprinati, D. (1995). An Introduction to the Theories of Popular Culture (2nd ed., pp. 88-96). London: Routledge.

Steinberg, M. (2001). Music and culture. Introduction. The Musical Quarterly, 85(3), 456-457. http://dx.doi.org/10.1093/mq/85.3.456

Warhol, A. (1962). Marilyn Diptych. London: Tate Modern.

Amlan Baisya is a Ph D Scholar in the Department of Humanities and Social Sciences, NIT Silchar. He has completed his B.A. in English Honours from Maulana Azad College, Kolkata, M.A. in English Literature from English and Foreign Languages University, Hyderabad and M.Phil in English Literature from Visva-Bharati University, Santiniketan. His research areas include Bangla Music tradition, Folk music and Bengali culture. (Orcid id: oooo-0oo2-5966-1108). Email: amlanb.1999@gmail.com

Dibyakusum Ray is an Assistant Professor in the Dept of Humanities and Social Sciences in NIT Silchar, Assam. He holds a Masters and an MPhil degree in English Literature and PhD in Commonwealth Literature from EFL University, Hyderabad. He has been a visiting scholar in TU Dresden and Assistant Professor in English in Symbiosis Law School, Hyderabad. Concerned with Continental Aesthetics, Liminality and Literature of Dissent, his current work is on Horror and Fantasy Literature, Madness and Post-millennial American Literature. (Orcid id: oooo-0oo2-95373277). Email: dibyakusum776@gmail.com 